\title{
Spin initialization of a p-doped quantum dot coupled to a bowtie nanoantenna
}

\author{
F. Carreño, Francisco Arrieta-Yáñez, M.A. Antón \\ Facultad de Óptica y Optometría, Universidad Complutense de Madrid, C/ Arcos de \\ Jalón 118, 28037 Madrid, Spain
}

\begin{abstract}
The spin initialization of a hybrid system consisting of a p-doped semiconductor quantum dot coupled to a gold bowtie nanoantenna is analyzed. The quantum dot is described as a four-level atom-like system using the density matrix formalism. The two lower levels are Zeeman-split hole spin states and the two upper levels correspond to positively charged excitons with a spin-up, spin-down hole pair and opposite spin electron. The gold bowtie nanoantenna is placed in close proximity to the quantum dot. A linearly polarized laser field drives two of the optical transitions of the quantum dot and produces localized surface charge oscillations in the nanoantenna which act back upon the quantum dot thus changing the effective field felt by it. The angular frequencies of those charge oscillations are very different along its two principal axes, resulting in an anisotropic modification of the spontaneous emission rates of the allowed optical transitions of the quantum dot. These changes are accounted for by using the Green tensor method, and result in a faster spin state initialization than that of the isolated quantum dot. We also show that the presence of the nanoantenna dramatically modifies the optical properties of the fluorescent photons, either in the spectral and in the time domain.
\end{abstract}

Keywords: Quantum Dot, Spin initialization, Gold, Plasmonics

Email address: ferpo@fis.ucm.es (F. Carreño) 


\section{Introduction}

The scalability, feasibility of coherent manipulation and strong robustness against relaxation make semiconductor quantum-dots (QDs) attractive as candidates to store quantum bits (qbits) [1, 2]. The resonant excitation of single QDs has enabled the experimental observation of Rabi oscillations [3], as well as the coherent manipulation of excitons [4] and the optical accessing of spins in QDs [5]. A well defined preparation of the initial quantum state is essential for quantum information processing, specially for computation and gate operations. The suppression of spin-orbit interaction of the electron spin has lead to consider it as a candidate for qbit. The bad news arise from the strong interaction with the reservoir of QD nuclei spin which limits the coherence to tens of ns [6]. The initialization of an electron spin state (optical pumping or laser cooling of an electron spin) has been experimentally demonstrated in a singly-charged QD using a magnetic field in the Faraday geometry [7]. An alternative to minimize the interaction of the spin with the reservoir of $\mathrm{QD}$ nuclei spin makes use of the confinement of a single hole spin to a QD. The p-type wave function of the hole has a node at the position of the QD nuclei with the consequent reduction in the hyperfine contact coupling Hamiltonian[8]. Brunner et al. [9] have reported a hole spin relaxation time in the millisecond scale. Recent progress on coherent spin pumping and repumping of a hole spin in a positively charged quantum dot in p-type $\delta$-doped QDs with a single laser has been achieved [10].

A fast state initialization speed, much faster than the quantum state decoherence rate, is also required for reliable quantum computation operations. Previous theoretical analysis have shown that a QD in the Voigt geometry allows for initialization times shorter than the typical spin coherence time accompanied by a reduction of the maximum attainable fidelity [11]. Loo et al. [12] have analyzed how the coupling of the QD with a high quality factor optical cavity results in a fast state initialization. The cavity system produces a strong modification of the local density of states which in turn produces a preferential, Purcell-accelerated de-excitation toward the desired final state, or target state. Antón et al [13] have proposed a system which combines both a p-type QD in the Voigt geometry and a cavity-like system to get a fast initialization of the spin state. A metallic nanoparticle (MNP) with ellipsoidal geometry was used to produce an anysotropic enhancement of the decay rates of the atomic transitions thus mimicking the cavity system and was shown to produce a fast and preferential de-excitation of the upper 
level to the target state.

It is well-known that metallic nanostructures can support resonant charge oscillations upon illumination with light of certain frequencies [14], the socalled surface plasmon resonances. The underlying physical mechanism relies on the fact that metallic nanoparticles with sizes smaller than the wavelength of light in vacuum support strong dipolar excitations, which are nonpropagating excitations of the conduction band electrons coupled to the light field [15]. These surface plasmons are tightly confined spatially, leading to a resonant modification of the local field felt by the QD [16, 17, 18, 19]. This effect has been explored either theoretically and from an experimental point of view in the context of the nonlinear enhancement of the second harmmonic generation in a hybrid system consisting of a quantum dot and a metallic nanoparticle [20]. In addition, the local density of states are dramatically altered by the nanoantennas, which results in a modification of the spontaneous emission rates of the QD's optical transitions [19, 21, 22, 23, 24]. The modifications of either linear and nonlinear optical properties of quantum systems placed in close proximity to a metallic nanostructure has been the subject of intense reasearch (see Refs. [25, 26, 27] for a recent although non extensive list of works on this topic). The plasmonic properties of a nanostructure depend dramatically on its size and shape, as has been demonstrated in studies of nanorods, [28, 29, 30] nanocubes, [31, 32] nanostars, 33] and numerous other structures [22]. Between these structures, single plasmonic dimers consisting of adjacent nanoparticle pairs, give rise to very large field corrections in their junctions, when the surface plasmons are excited [34] which make them highly attractive as SERS substrates, with enhancements approaching single-molecule sensitivity. Plasmonic dimers have been widely studied due to the tunability of their far-field scattering properties in the visible/NIR range [35], and strong coupling between individual nanostructured plasmonic dimers and J-aggregate complexes has also been studied [36]. Graphene nano-bowties has been shown to support localized plasmons with unprecedented levels of field confinement [37, 22].

In this work we propose a scheme to obtain a fast hole spin initialization with high fidelity in a hybrid system consisting of a p-doped QD and a gold bowtie nanoantenna. We will consider the so-called Voigt configuration in which the magnetic field is applied along the growth plane of the QD. We present numerical simulations for a representative case of $\mathrm{QD}$, with values of the oscillator strengths, decay rates and the characteristic energy levels taken from experimental studies [9]. The Green tensor method is used to account 
for the modification of the decay rates and the local field correction. We show that a proper adjustment of the shape and separation of the nanoantenna (the gap distance), and the Rabi frequency of the driving field allows for a faster spin initialization than for the isolated QD. In addition, we present a new method to estimate whether the spin has been effectively initialized, the so-called characteristic time $\left(T_{0}\right)$, which differs from previous methods used to estimate such instants of time [11, 38]. The current proposal to estimate $T_{0}$ is more robust than that previously used, and allows for the specification of an interval of Rabi frequencies which should produce the spin initialization of the QD.

The paper is organized as follows: Section 2 establishes the model, i.e. the Hamiltonian of the system and the time-evolution equations of the atomic operators assuming the rotating wave approximation incorporating the effects of field correction and the modification of the decay rates by making use of the Green tensor method. Section 3 deals with the numerical simulations and explores the possibility of obtaining fast spin initialization via the plasmonic interaction. Section 4 deals with the plasmonic effects on the statistics of the fluorescent field: we describe the spectral properties of the fluorescent photons through the analysis of the resonance fluorescence spectrum, and the temporal properties of the second-order correlation functions of such fluorescent photons. Finally, section 5 summarizes the main conclusions.

\section{Theoretical model}

We consider an InAs/GaAs self-assembled QDs via the Stranski-Krastanov method with growth direction along the $Z$-axis. The QDs are separated from a Fermi sea of holes by several nanometers in thick p-doped back contact layer. An external bias voltage applied between the top gate and the back contact allows the control of the QD charge state [39, 40]. The ground hole states are labeled $\left|1_{z}\right\rangle \equiv|\Downarrow\rangle$ and $\left|2_{z}\right\rangle \equiv|\Uparrow\rangle$, while the excited trion states are $\left|3_{z}\right\rangle \equiv|\Downarrow \Uparrow \uparrow\rangle$ and $\left|4_{z}\right\rangle \equiv|\Downarrow \Uparrow \downarrow\rangle$. Here $\Uparrow(\Downarrow)$ and $\uparrow(\downarrow)$ denote a heavy hole $(\mathrm{HH})$ and an electron with spins along (against) the $Z$-axis. Hole and electron spin states share the same energy level in the absence of an external magnetic field. The energy level diagram of such kind of QD is depicted in Fig. 1(a). The optical transition $\left|1_{z}\right\rangle \leftrightarrow\left|4_{z}\right\rangle /\left|2_{z}\right\rangle \leftrightarrow\left|3_{z}\right\rangle$ is driven by a $\sigma^{+} / \sigma^{-}$polarized laser field, while due to selection rules transitions $\left|2_{z}\right\rangle \leftrightarrow\left|4_{z}\right\rangle$ and $\left|1_{z}\right\rangle \leftrightarrow\left|3_{z}\right\rangle$ remain dark. The application of an external magnetic field along the $X$-axis, in the so-called Voigt geometry, results in that dark transi- 
tions become bright. The magnetic field lifts the degeneracy of hole/electron levels according to $E_{Z m}^{h(e)}=\frac{1}{2} \mu_{B} g^{h(e)} B_{x}$, where $E_{Z m}^{h(e)}$ stands for the Zeeman energy shift relative to $B_{x}=0 \mathrm{~T}, B_{x}$ being the external magnetic field, and $\mu_{B}$ is the Bohr magneton. The quantity $g^{h(e)}$ is the Landé factor of carrier $h(e)$. In the $X$-basis the ground hole states are $|1\rangle \equiv\left|\Downarrow_{x}\right\rangle=\frac{1}{\sqrt{2}}(|\Downarrow\rangle-|\Uparrow\rangle)$ and $|2\rangle \equiv\left|\Uparrow_{x}\right\rangle=\frac{1}{\sqrt{2}}(|\Downarrow\rangle+|\Uparrow\rangle)$. In addition, the electron spin states are $\left|\uparrow_{x}\right\rangle=\frac{1}{\sqrt{2}}(|\uparrow\rangle+|\downarrow\rangle)$ and $\left|\downarrow_{x}\right\rangle=\frac{1}{\sqrt{2}}(|\uparrow\rangle-|\downarrow\rangle)$, while the mixed trion states are $|4\rangle=\left|\Uparrow_{x} \Downarrow_{x} \downarrow_{x}\right\rangle$, and $|3\rangle=\left|\Uparrow_{x} \Downarrow_{x} \uparrow_{x}\right\rangle$. The resulting selection rules in the $X$-basis now read

$$
\begin{aligned}
& \left\langle 4\left|E_{x}\right| 1\right\rangle \neq 0,\left\langle 4\left|E_{y}\right| 1\right\rangle=0, \\
& \left\langle 3\left|E_{x}\right| 2\right\rangle \neq 0,\left\langle 3\left|E_{y}\right| 2\right\rangle=0, \\
& \left\langle 4\left|E_{x}\right| 2\right\rangle=0,\left\langle 4\left|E_{y}\right| 2\right\rangle \neq 0, \\
& \left\langle 3\left|E_{x}\right| 1\right\rangle=0,\left\langle 3\left|E_{y}\right| 1\right\rangle \neq 0 .
\end{aligned}
$$

Here,

$$
\begin{aligned}
& E_{x}=\frac{1}{2}\left(\sigma^{+}+\sigma^{-}\right), \\
& E_{y}=\frac{1}{2}\left(\sigma^{+}-\sigma^{-}\right),
\end{aligned}
$$

was used. Now each hole spin ground state can be linked to two exciton states via linearly and orthogonally polarized transitions. The four levels of the system in the Voigt configuration are depicted in Fig. 1(b). The bowtie nanoantenna can be considered like a continuum of states which is resonant with the trion states. Similar arrangements have been previously considered in other works [34, 41, 42, 43].

The Hamiltonian that governs the dynamics of the coupled QD-nanoantenna hybrid system can be expressed in the rotating-wave approximation as

$$
H=H_{F}+H_{A}+H_{\text {Int }}+H_{E x t} .
$$

$H_{F}$ is the Hamiltonian of the medium-assisted electromagnetic field

$$
H_{F}=\int d \vec{r} \int_{0}^{\infty} d \omega \hbar \omega \vec{f}_{\lambda}^{\dagger}(\vec{r}, \omega) \vec{f}_{\lambda}(\vec{r}, \omega)
$$

which is expressed in terms of a set of bosonic fields $\vec{f}(\vec{r}, \omega)$ and $\vec{f}(\vec{r}, \omega)$ plays the role of variable of the electromagnetic field and the medium, including a 

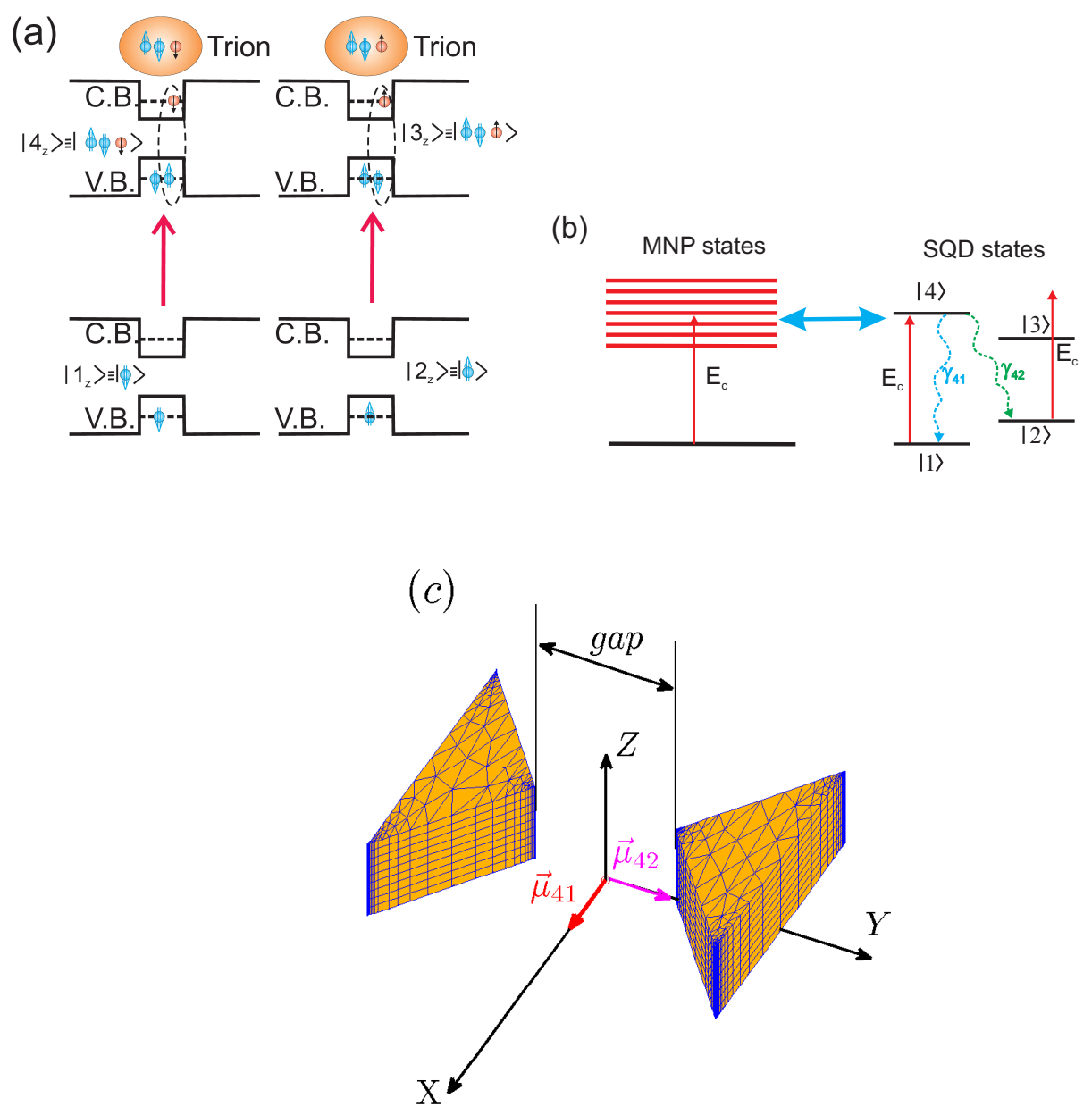

Figure 1: (a) Four level scheme illustrating the ground and excited states of self-assembled QDs. C.B./V.B. stands for the Conduction/Valence Band. (b) Energy levels of the QD in the Voigt geometry and the nanoantenna. The QD atomic states in the $X$ basis are $|1\rangle$ and $|2\rangle$ for the hole spin eigenvectors, split by the Zeeman effect; and the upper levels $|3\rangle$ and $|4\rangle$ are $X^{1+}$ excitons consisting of two spin-paired holes in the V.B. and an unpaired electron with spin $\pm 1 / 2$ in the C.B. The straight lines indicate the driving field linearly polarized along the $X$ axis. The wavy lines indicate the spontaneous relaxations from the upper level $|4\rangle$ to the ground states. In addition, $\gamma_{32}$ and $\gamma_{31}$ are the decay rate of the upper level $|3\rangle$ to the ground states transitions (not shown). (c) Bowtie nanoantenna separated by a distance gap along the $Y$ axis. The computational grid of the nanoantenna is also shown. 
reservoir associated with the losses in the medium. The field operators obey the usual commutation rules

$$
\left[\vec{f}(\vec{r}, \omega), \overrightarrow{f^{\dagger}}(\vec{r}, \omega)\right]=\delta\left(\omega-\omega^{\prime}\right) \delta\left(\vec{r}-\overrightarrow{r^{\prime}}\right) .
$$

The free Hamiltonian $H_{A}$ of the four-level QD system read as

$$
H_{A}=\sum_{j=1}^{4} \hbar \omega_{j} \hat{\sigma}_{j j}
$$

where $E_{n}=\hbar \omega_{j}$, are the energies of the QD and $\sigma_{i j}$ are the Pauli operators of the excitation electron-hole pair. The interaction Hamiltonian is $H_{\text {int }}$

$$
H_{\text {Int }}=-\int_{0}^{\infty} d \omega[\vec{d} \cdot \vec{E}(\vec{r}, \omega)+H . c .]
$$

$\vec{d}$ being the atomic dipole moment operator, which is given by

$$
\vec{d}=\vec{\mu}_{14} \hat{\sigma}_{14}+\vec{\mu}_{24} \hat{\sigma}_{24}+\vec{\mu}_{23} \hat{\sigma}_{23}+\vec{\mu}_{13} \hat{\sigma}_{13}
$$

and $\vec{E}(\vec{r}, \omega)$ is the field operator (excluding the external driving field) which is defined through [44]

$$
\vec{E}(\vec{r}, \omega)=i \sqrt{\frac{\hbar}{\pi \epsilon_{0}}} \frac{\omega^{2}}{c^{2}} \int d \overrightarrow{r^{\prime}} \sqrt{\epsilon_{I}\left(\overrightarrow{r^{\prime}}, \omega\right)} \stackrel{\leftrightarrow}{G}\left(\vec{r}, \overrightarrow{r^{\prime}}, \omega\right) \vec{f}\left(\overrightarrow{r^{\prime}}, \omega\right) .
$$

In Eq. (9) $\stackrel{\leftrightarrow}{G}\left(\vec{r}, \overrightarrow{r^{\prime}}, \omega\right)$ is the dyadic Green's tensor which is a solution of the Helmholtz equation

$$
\left[\nabla \times \nabla \times-\frac{\omega^{2}}{c^{2}} \epsilon(\vec{r}, \omega)\right] \stackrel{\leftrightarrow}{G}\left(\vec{r} \overrightarrow{r^{\prime}} \omega\right)=\stackrel{\leftrightarrow}{I} \delta\left(\vec{r}-\overrightarrow{r^{\prime}}\right),
$$

together with the boundary condition $\stackrel{\leftrightarrow}{G}\left(\vec{r}, \overrightarrow{r^{\prime}}, \omega\right) \rightarrow 0$ for $\left|\vec{r}-\overrightarrow{r^{\prime}}\right| \rightarrow \infty$, and $\overleftrightarrow{I}$ being the unit dyadic. In addition, the Green's tensor obeys the useful integral relation,

$$
\int d^{3} s \frac{\omega^{2}}{c^{2}} \epsilon_{I}(s, \omega) \stackrel{\leftrightarrow}{G}(\vec{r}, s, \omega) \stackrel{\leftrightarrow}{G^{*}}\left(s, \vec{r}^{\prime}, \omega\right)=\operatorname{Im}\left[\stackrel{\leftrightarrow}{G}\left(\vec{r}, \overrightarrow{r^{\prime}}, \omega\right)\right]
$$


which follow directly from the Helmholtz equation [44]. Here, $\epsilon\left(\overrightarrow{r^{\prime}}, \omega\right)=$ $\epsilon_{R}\left(\overrightarrow{r^{\prime}}, \omega\right)+\epsilon_{I}\left(\overrightarrow{r^{\prime}}, \omega\right)$ stands for the complex permittivity.

Finally $H_{E x t}$ is the part of the Hamiltonian which accounts for the external coherent pumping and is defined through

$$
H_{E x t}=\hbar\left(\Omega_{41} \sigma_{41}+\Omega_{32} \sigma_{32}\right) e^{-i \omega_{L} t}+\text { H.c. },
$$

with the effective Rabi field $\Omega_{i j}=\vec{\mu}_{i j} \cdot \vec{E}_{\text {pump }}\left(\vec{r}_{A}\right) / 2 \hbar, \vec{r}_{A}$ being the position of the QD in relation to the nanoantenna $\left(R=\left|\vec{r}_{A}\right|\right)$. The pump field contains the direct pumping field term plus the scattered field from the MNP

$\vec{E}_{\text {pump }}\left(\vec{r}_{A}, \omega_{L}\right)=\vec{E}_{0}\left(\vec{r}_{A}, \omega_{L}\right)+\int_{V_{M N P}} d \overrightarrow{r^{\prime}}\left(\epsilon_{M N P}\left(\omega_{L}\right)-1\right) \stackrel{\leftrightarrow}{G}\left(\vec{r}_{A}, \overrightarrow{r^{\prime}}, \omega_{L}\right) \vec{E}_{0}\left(\overrightarrow{r^{\prime}}, \omega_{\mathbb{Z}} \beta\right)$

where $\vec{E}_{0}\left(\vec{r}_{A}, \omega_{L}\right)=\frac{1}{2} \hat{u}_{x} E_{0} e^{-i \omega_{L} t}+$ c.c. is the incident field operator and $\hat{u}_{x}$ is the unitary vector along the $X$-axis, and $\epsilon_{M N P}\left(\omega_{L}\right) / V_{M N P}$ stands for the dielectric constant/volume of the bowtie at the frequency $\omega_{L}$. Therefore, the driving field only couples transitions $|1\rangle \leftrightarrow|4\rangle$ and $|2\rangle \leftrightarrow|3\rangle$. Note that for an intense incident driving field $\left(\vec{E}_{0}\left(\vec{r}, \omega_{L}\right)\right)$ it can be treated as a c-number, so that the effective Rabi field can be expressed as

$$
\Omega_{i j}=\Omega_{c}^{0}\left[1+\int_{V_{M N P}} d \overrightarrow{r^{\prime}}\left(\epsilon_{M N P}\left(\omega_{L}\right)-1\right) \hat{u}_{x} \stackrel{\leftrightarrow}{G}\left(\vec{r}_{A}, \vec{r}^{\prime}, \omega_{L}\right) \hat{u}_{x}\right] \equiv \Omega_{c}^{0} F_{e}(i=1,2, j=4(
$$

$F_{e}$ being the field enhancement factor. In the above equation $\Omega_{i j}$ is the renormalized Rabi frequency associated with the driving field and the field produced by the nanoantenna which acts back upon the QD, and $\Omega_{c}^{0} \equiv \frac{\mu_{i j} E_{0}}{2 \hbar}$ stands for the free spece Rabi frequency, i.e., the Rabi frequency which would drive the QD transitions in the absence of the bowtie.

In this work we are interested in the dynamical evolution of the QD, so we only need the reduced density matrix of the system. For this purpose we shall derive the master equation by employing the Hamiltonian in Eq. (3) and the Heisenberg picture. Thus the time evolution of an arbitrary atomic operator $Q(t)$ is governed by the Heisenberg equation of motion

$$
\frac{\partial<Q(t)>}{\partial t}=\frac{i}{\hbar}\left[H_{A}+H_{E x t}, Q\right]+\frac{i}{\hbar}\left[H_{i n t}, Q\right] .
$$


The equation for the field operator $\vec{f}(\vec{r}, \omega)$ is given by

$$
\begin{aligned}
\frac{\partial \vec{f}}{\partial t}= & -i \omega \vec{f}+\frac{i}{\hbar}\left[\vec{\mu}_{41} \stackrel{\leftrightarrow}{G}^{*}\left(\vec{r}_{A}, \vec{r}, \omega\right) \sigma_{14}+\vec{\mu}_{42} \stackrel{\leftrightarrow}{G}^{*}\left(\vec{r}_{A}, \vec{r}, \omega\right) \sigma_{24}\right. \\
& \left.+\vec{\mu}_{31} \stackrel{\leftrightarrow}{G}^{*}\left(\vec{r}_{A}, \vec{r}, \omega\right) \sigma_{13}+\vec{\mu}_{32} \stackrel{\leftrightarrow}{G}^{*}\left(\vec{r}_{A}, \vec{r}, \omega\right) \sigma_{23}\right] .
\end{aligned}
$$

We formally integate Eq. (16) and then we insert back into Eq.(15) by assuming the leading order in the coupling, so the equation of motion for the atomic variables of the medium yields

$$
\begin{aligned}
\frac{\partial<Q(t)>}{\partial t}= & \frac{i}{\hbar}\left[H_{A}+H_{E x t}, Q\right]-\frac{\gamma_{41}^{p}}{2}\left(\sigma_{41}\left[\sigma_{14}, Q\right]-\left[\sigma_{41}, Q\right] \sigma_{14}\right) \\
& -\frac{\gamma_{42}^{p}}{2}\left(\sigma_{42}\left[\sigma_{24}, Q\right]-\left[\sigma_{42}, Q\right] \sigma_{24}\right) \\
& -\frac{\gamma_{31}^{p}}{2}\left(\sigma_{31}\left[\sigma_{13}, Q\right]-\left[\sigma_{31}, Q\right] \sigma_{13}\right) \\
& -\frac{\gamma_{32}^{p}}{2}\left(\sigma_{32}\left[\sigma_{23}, Q\right]-\left[\sigma_{32}, Q\right] \sigma_{23}\right) \\
& -\frac{\gamma_{21}}{2}\left(\sigma_{21}\left[\sigma_{12}, Q\right]-\left[\sigma_{21}, Q\right] \sigma_{12}\right) .
\end{aligned}
$$

Here, $\gamma_{i j}^{p}$ stands for the spontaneous emission of the SQD modified by the presence of the nanoantenna [45, 46, 47, 48, 49] which are explicitely given by

$$
\begin{aligned}
& \gamma_{32}^{p} \approx \gamma_{41}^{p}=\frac{\mu_{41}^{2} \omega_{41}^{2}}{c^{2} \hbar \epsilon_{0}} \hat{u}_{x} \cdot \operatorname{Im}\left[\stackrel{\leftrightarrow}{G}\left(\vec{r}_{A}, \vec{r}_{A}, \omega_{41}\right)\right] \cdot \hat{u}_{x} \equiv \gamma_{41}^{(0)} F_{e n h}^{x} \\
& \gamma_{31}^{p} \approx \gamma_{42}^{p}=\frac{\mu_{41}^{2} \omega_{42}^{2}}{c^{2} \hbar \epsilon_{0}} \hat{u}_{y} \cdot \operatorname{Im}\left[\stackrel{\leftrightarrow}{G}\left(\vec{r}_{A}, \vec{r}_{A}, \omega_{42}\right)\right] \cdot \hat{u}_{y} \equiv \gamma_{41}^{(0)} F_{e n h}^{y}
\end{aligned}
$$

where $\hat{u}_{y}$ stands for a unit vector along the $Y$ axis, and superindex 0 is used to indicate the values of the decay rates in free space. In addition, $\stackrel{\leftrightarrow}{G}\left(\vec{r}_{A}, \vec{r}_{A}, \omega_{41}\right)$ is the electromagnetic Green's tensor evaluated at the frequency $\omega_{41} . F_{e n h}^{x / y}$ stands for the plasmonic enhancement of the radiative decay rates along $X / Y$ direction. These parameters account for the plasmonic field of the nanoantenna. The dissipative process described by the term with the pre-factor $\gamma_{21}$ accounts for the lower levels' dephasing and is assumed to be uncoupled with the localized surface plasmons due to the low values of the Zeeman splitting considered. It is worth noting that depending on the orientation of the dipole 
moments of the QD emitter's transitions, the decay rates could become very different from one another, i.e., the value of $\gamma_{41}^{p}$ can strongly differ from that of $\gamma_{42}^{p}$. This will result in an anisotropic Purcell factor enhancement which will have important consequences for controlling the time dynamics of this nano-hybrid system, and can lead for example to an acceleration or deceleration of the spin initialization to the selected state [13]. The decay rate $\gamma_{j k}^{p}(j=3,4, k=1,2)$ accounts for all processes that provide dissipative transitions between the discrete levels of the QD. The different contributions to $\gamma_{j k}^{p}$ may be written as

$$
\gamma_{j k}^{p}=\gamma_{j k}^{p, r a d}+\gamma_{j k}^{p, \text { nonrad }} .
$$

Emission into free space radiating electromagnetic modes is labeled by $\gamma_{j k}^{p, r a d}$. Furthermore, another emitter lossy channel specific to plasmonic structures arises when the emitter is placed close to a MNP, resulting in the coupling of the emitter to nonpropagating, quickly decaying evanescent modes where the energy is dissipated through heating in the metal. The associated rate is called $\gamma_{j k}^{p, n o n r a d}$ and can assume very high rates when the emitter is closed to the metal surface. In fact, $\gamma_{j k}^{p \text {,nonrad }}$ is the dominant contribution to the total decay rate at emitter-metal distances below $\approx 10 \mathrm{~nm}[50]$. To avoid this quenching effect, one may lift the QD away from the metal surface by placing it at an intermediate region close enough to still couple efficiently to the localized surface plasmons.

From Eq. (17), we obtain the following equations of motion for the density 
matrix elements of the QD of the hybrid system:

$$
\begin{aligned}
\frac{\partial \rho_{41}}{\partial t} & =-\left[\Gamma_{41}-i \Delta_{c}\right] \rho_{41}+i \Omega_{c} \rho_{21}+i \Omega_{c}\left(\rho_{11}-\rho_{44}\right), \\
\frac{\partial \rho_{31}}{\partial t} & =-\left[\Gamma_{31}-i\left(\Delta_{c}+2 \Delta_{e}\right)\right] \rho_{31}+i \Omega_{c} \rho_{21}-i \Omega_{c} \rho_{34}, \\
\frac{\partial \rho_{21}}{\partial t} & =-\left[\Gamma_{21}+i \omega_{21}\right] \rho_{21}+i \Omega_{c}^{*} \rho_{31}-i \Omega_{c} \rho_{24}, \\
\frac{\partial \rho_{43}}{\partial t} & =-\left[\Gamma_{43}+i 2 \Delta_{c}\right] \rho_{43}+i \Omega_{c} \rho_{13}-i \Omega_{c}^{*} \rho_{42}, \\
\frac{\partial \rho_{42}}{\partial t} & =-\left[\Gamma_{42}-i\left(\Delta_{c}+2 \Delta_{g}\right)\right] \rho_{42}+i \Omega_{c} \rho_{12}-i \Omega_{c} \rho_{43}, \\
\frac{\partial \rho_{32}}{\partial t} & =-\left[\Gamma_{32}-i\left(\Delta_{c}+2 \Delta_{e}+2 \Delta_{g}\right)\right] \rho_{32}+i \Omega_{c}\left(\rho_{22}-\rho_{33}\right) \\
\frac{\partial \rho_{44}}{\partial t} & =-\left(\gamma_{41}^{p}+\gamma_{42}^{p}\right) \rho_{44}+i \Omega_{c} \rho_{14}-i \Omega_{c}^{*} \rho_{41}, \\
\frac{\partial \rho_{33}}{\partial t} & =-\left(\gamma_{31}^{p}+\gamma_{32}^{p}\right) \rho_{33}+i \Omega_{c} \rho_{23}-i \Omega_{c}^{*} \rho_{32}, \\
\frac{\partial \rho_{22}}{\partial t} & =-\gamma_{21} \rho_{22}+\gamma_{32}^{p} \rho_{33}+\gamma_{42}^{p} \rho_{44}+i \Omega_{c}^{*} \rho_{32}-i \Omega_{c} \rho_{23} .
\end{aligned}
$$

To abtain the above equations we have assumed that $\Omega_{41}=\Omega_{32} \equiv \Omega_{c}$ with the following definition for dephasings: $\Gamma_{41}=\left(\gamma_{41}^{p}+\gamma_{42}^{p}\right) / 2, \Gamma_{21}=\gamma_{21} / 2$, $\Gamma_{32}=\left(\gamma_{31}^{p}+\gamma_{32}^{p}+\gamma_{21}\right) / 2=\Gamma_{41}+\Gamma_{21} .2 \Delta_{e}=E_{Z m}^{e}$, and $2 \Delta_{g}=E_{Z m}^{h}$. Finally, $\Delta_{c}=\omega_{L}-\omega_{41}$ denotes the optical detuning of the driving field. The population of the ground level $\rho_{11}$ is computed assuming a closed system, i.e., $\rho_{11}=1-\rho_{22}-\rho_{33}-\rho_{44}$.

A close inspection of Eq. (21) reveals that the plasmonic interaction manifests in two ways: The first relies on the plasmon-induced modification of the spontaneous decay rates. The second is related with the enhacement of the Rabi frequency which drives the QD according to the expression given in Eq. (14). The above mentioned mechanisms strongly depend on the QD-nanoantenna distance $R$. These mechanisms have been explored in the context of obtaining hole spin initialization [13] and in the case of considering the modification in the resonance fluorescence spectrum arising from the plasmonic interaction [38]. 


\section{Spin initialization}

As a first step in our study we focus our attention on the modifications of the spontaneous emission rates due to the presence of the MNP. We consider the data for the QD reported in Ref. [9]. In particular, the free space radiative decay rates of the transitions are $\hbar \gamma_{41}^{(0)}=\hbar \gamma_{42}^{(0)}=\hbar \gamma_{32}^{(0)}=\hbar \gamma_{31}^{(0)} \equiv$ $\hbar \gamma_{0}=0.5 \mu \mathrm{eV}$, the hole spin decay rate is $\gamma_{21} \simeq 0.000671 \mu \mathrm{eV}$, and the ground-level Zeeman splitting is $2 \hbar \Delta_{g}=36 \mu \mathrm{eV}$. These data correspond [9] to a temperature of $4.2 \mathrm{~K}$ and a magnetic field of $4.6 \mathrm{~T}$. We assume an upper value for the Zeeman splitting $2 \hbar \Delta_{e} \simeq 20 \mu \mathrm{eV}$. We also consider the driving field resonant with transition $|1\rangle \leftrightarrow|4\rangle$, i.e., $\Delta_{c}=0(\lambda \simeq 947 \mathrm{~nm})$. The MNP is a gold bowtie nanoantenna symetrically located between the QD (see Fig. 1(c)). The nanoantenna is made upon two equilateral triangles 135 $\mathrm{nm}$ in length and $35 \mathrm{~nm}$ in height. In addition, the dielectric constant of the host material is set to $\epsilon_{B}=2.25$.
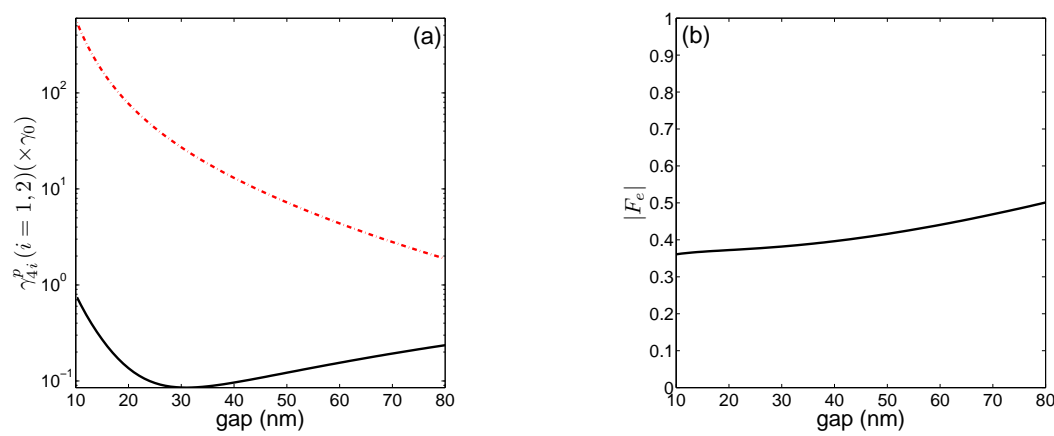

Figure 2: (a) Plasmon modified radiative decay rates of the $\mathrm{QD} \gamma_{42}^{p}$ (dashed-dotted line), and $\gamma_{41}^{p}$ (solid line) in units of $\gamma_{0}$ versus the gap between the two elements of the nanoantenna. (b) Absolute value of the field correction of the Rabi frequency of the driving field $\Omega_{c}\left(\left|F_{e}\right|\right)$ as a function of the gap distance.

Figure 2(a) show the radiative decay rates $\gamma_{42}^{p}$ and $\gamma_{41}^{p}$ modified by the presence of the MNP as a function of the gap distance between the two elements of the nanoantenna. The numerical results have been obtained by using the procedure described in Ref. [51]: in this computational approach, the surface of the MNP is discretized by a set of triangles and the electromagnetic potentials are matched at the triangle centers. By fullfilling the boundary conditions imposed by Maxwell's equations through auxiliary surface charges and currents, we can estimate the optical properties of the MNP 
with the selected shape embedded in a dielectric environment as depicted in Fig. 1(c). The dielectric function of the gold MNP was extracted from optical data [52] and the chosen mesh sizes allow for a spatial resolution of approximately $1 \mathrm{~nm}$. A close inspection of Fig. 2(a) shows a highly distinctive behaviour for the two decay rates: The $\gamma_{41}^{p}$ decay rate shows a monotonous and smooth variation versus the gap distance around the free space value $\left(\gamma_{0}\right)$, whereas the $\gamma_{42}^{p}$ decay rate exhibits a large variation taking values up to two orders of magnitude greater than the free space value. This asymmetric behavior is of great interest since it will allow to privilege the decay from the upper state $|4\rangle$ to the lower state $|2\rangle$ in comparison with the decay from $|4\rangle$ to the lower state $|1\rangle$. This behavior has its origin in the difference between the longitudinal surface plasmon resonance along the $Y$-axis and the transverse surface plasmon resonance along the $X$-axis. This indicates that the nanoantenna acts as a nanoscale cavity which enhances the strength of the vacuum fluctuations depending on the gap distance and the orientation of the dipoles of the system. Figure 2(b) depicts the absolute value of the plasmon modified Rabi frequency of the driven transition $|1\rangle \leftrightarrow|4\rangle$ versus the gap distance, revealing that such field correction remains above unity for all the distances considered. We conclude that a proper selection of the gap distance between the nanoantenna's elements can be used to enhance on demand the spontaneous emission in a selected atomic transition of the QD while modifying the Rabi frequency effectively felt by the QD. This anisotropic resonant phenomenon is the key idea that allows the selective plasmonic acceleration of the decay of the transition $|2\rangle \leftrightarrow|4\rangle$ in comparison with the almost unaltered transition $|1\rangle \leftrightarrow|4\rangle$. We will show that this will result in dramatic modifications of the initialization time of the spin.

After having studied the effect of the nanoantenna on the spontaneous emission, now we turn our attention to its impact on the time dynamics of the hybrid system, and in particular, in the time required for the initialization of the spin. To this end we have carried out numerical simulations by solving the density matrix equations [Eq. (21)], using a time interval ranging from $t_{0}=0$ to $t_{F}=50 \gamma_{0}^{-1}$, where the spontaneous decay time is $\gamma_{0}^{-1} \simeq 1.3 \mathrm{~ns}$. This time interval allows us to guarantee that steady-state is reached. We also consider standard atomic initial conditions given by $\rho_{11}(0)=1$, and the rest of elements of $\rho$ are set to zero. In particular, we are interested in the initialization of the spin sate in the QD. The spin state preparation consists of the resonant excitation of $|1\rangle \rightarrow|4\rangle$ transition by the $X$-polarized field. Then, the system can relax to the desired state $|2\rangle$ or to the initial state $|1\rangle$ 

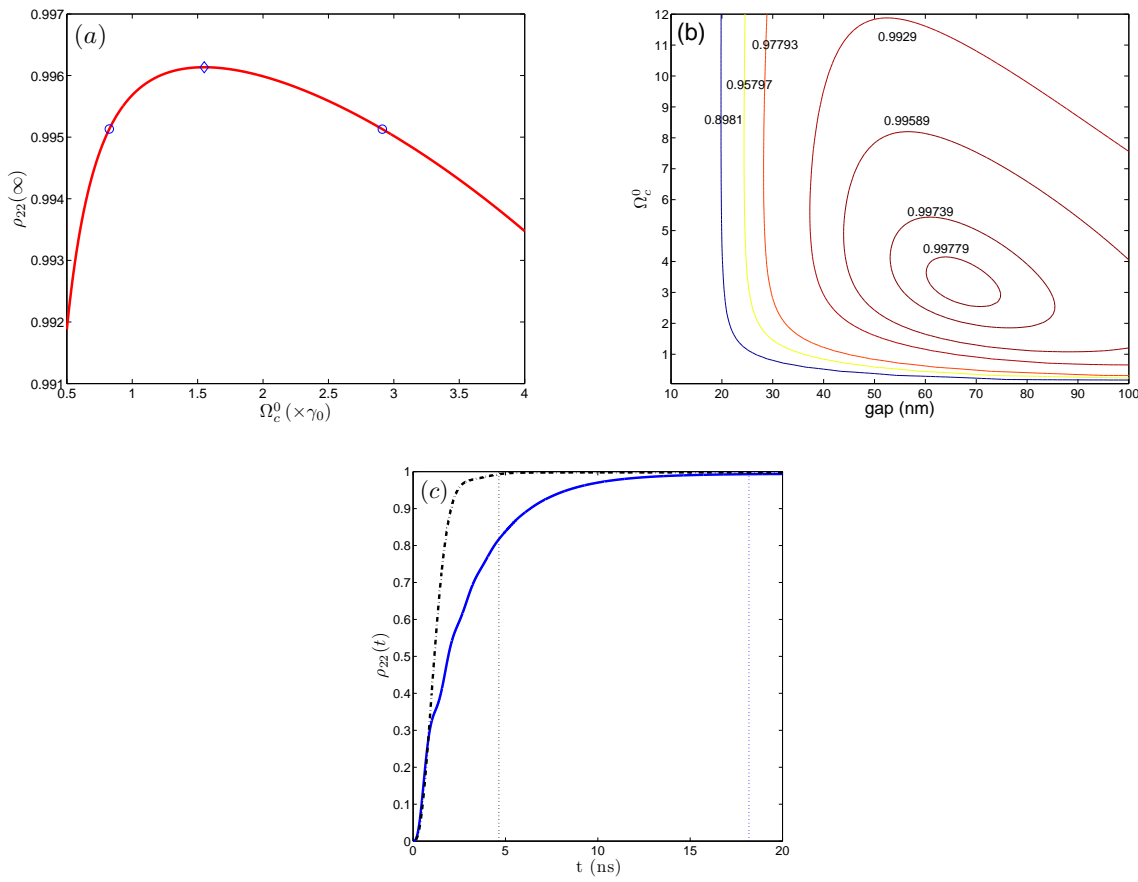

Figure 3: (a) Steady state population of the target state $|2\rangle\left(\rho_{22}(\infty)\right)$ for the isolated QD versus the Rabi frequency $\Omega_{c}^{0}$. (b) Contour plot of the Steady state population of the target state $|2\rangle$ versus the gap distance between the nanoantenna's elements and the Rabi frequency $\Omega_{c}^{0}$. (c) Time evolution of the population of the target state $\rho_{22}(t)$ for $\Omega_{c}^{0}=3.5 \gamma_{0}$ in the case of an isolated QD (solid line) and for the hybrid system (dashed-dotted line) with a gap distance of $65 \mathrm{~nm}$.

at different rates $\left(\gamma_{42}^{p} \neq \gamma_{41}^{p}\right)$. The population of the target state $|2\rangle$ increases with a characteristic time $T_{0}$ which is usually related to the spontaneous decay rate of the exciton.

Let us start by considering the population of the target state $|2\rangle$ for the isolated QD. Figure 3(a) displays the steady state population $\left(\rho_{22}(\infty)\right)$ achieved versus the Rabi frequency of the driving field $\Omega_{c}^{0}$. There we can appreciate the existence of an optimum Rabi frequency $\left(\Omega_{c}^{0, o p t}=1.55 \gamma_{0}\right)$ which produces the maximum transfer of population to state $|2\rangle\left(\rho_{22}(\infty)=\right.$ 0.9961). This point is labeled with a blue diamond. In what follows we will assume that the spin of the system is effectively initialized when the population has reached a certain prescribed value within a certain mismatch or tolerance, $T O L$, let us say for example that $T O L=0.001$. In particular, 
in the case of driving the system with $\Omega_{c}^{0, o p t}$ we will assume that the spin has been initialized when the population $\rho_{22}\left(t=T_{0}\right)$ has reached a value of $\rho_{22}\left(t=T_{0}\right)=0.9961-0.001=0.9951$. The time elapsed to reach such population, $T_{0}$, will be termed as to the characteristic time. This definition of the characteristic time differs from the one previously used by other auhtors [11, 34, 12, 13] which was based on computing the real part of the smallest eigenvalue of the set of Eqs. (21) in the weak-field limit. This resulted in predicting initialization times $T_{0}$ smaller tha $\gamma_{0}^{-1}$, although the level of population achieved at such instant of time is far from its corresponding steady-state value, thus at such instant of time the system is far from be effectively initialized. By using the definition of $T_{0}$ proposed in this work, we are certanly aware that the system has reached the level of population previously prescribed within a certain tolerance, and it becomes a robust definition for such "characteristic time". In addition, by using such tolerance we can determine an interval of Rabi frequencies of the driving field which allow to initialize the system. In the current case the endpoints of such interval are depicted in Fig. 3(a) with blue circles and the interval results to be $\left[0.825 \gamma_{0}, 2.912 \gamma_{0}\right]$.

Figure 3(b) shows a contour plot of the steady state population of the target state $\left(\rho_{22}(\infty)\right)$ versus the gap distance and the free space Rabi frequency $\left(\Omega_{c}^{0}\right)$ of the driving field. Note that the maximum transfer of population achieved is 0.9978 which is sligthly larger than that previously obtained for the case of the isolated QD. The optimum values for the gap and the Rabi frequencies are $67 \mathrm{~nm}$ and $3.37 \gamma_{0}$, respectively. This plot reveals the existence of an interval of Rabi frequencies $\left(\left[2.5 \gamma_{0}, 4 \gamma_{0}\right]\right)$ and an interval of gap distances $([60,75] \mathrm{nm})$ which results in an optimum transfer of population for the particular size and shape of nanoantenna selected.

With the previous results in mind we are now in conditions to determine how the presence of the nanoantenna influences the time dynamics of the system. We have solved Eq. (21) to obtain the time evolution of population and coherences for a Rabi frequency of the driving field belonging to the interval mentioned in the last paragraph. The results are depicted in Fig. 3(c). This plot has been produced for $\Omega_{c}^{0}=3.5 \gamma_{0}$ and a gap distance of $65 \mathrm{~nm}$, close to the optimum values previously mentioned for the hybrid system, and results in $\gamma_{41}^{p}=0.17 \gamma_{0}$, and $\gamma_{42}^{p}=3.48 \gamma_{0}$, while $\left|F_{e}\right|=0.45$. The solid line corresponds to the result obtained for the isolated QD whereas the dashed-dotted line corresponds to the hybrid system. Numerical analysis reveals that the values for $T_{0}$ obtained for the isolated QD is 18.18 ns whereas 
it reduces to $4.64 \mathrm{~ns}$ in the case with the nanoantenna. These characteristic times were determined by setting the prescribed level of population in the target state to be 0.9942 with a tolerance $T O L=0.001$. These results clearly indicate that the plasmonic interaction produces a threefold acceleration of the spin initialization. It is worth mentioning that the characteristic times determined using the procedure described in Ref. [13] are $2.68 \mathrm{~ns}$ and 0.65 ns for the isolated QD and the hybrid system, respectively. However, a close inspection of Fig. 3(c) reveals that at those instants of time the system is far from be initialized, as previously indicated.

\section{Plasmonic effects on the photon statistics of the fluorescent field}

Quantum optical experiments allow also to investigate the statistics of emitted light. Previously Ridolfo et al. [53] have investigated theoretically the photon/plasmon statistics of one SQD and one MNP. They showed a strong impact of the interaction between the SQD and theMNP on the statistics of the light emitted by the interacting hybrid system. In this section we extend the previously investigated hybrid structure and include the interaction between a metallic dimer coupled to one QD.

First, we proceed to analyze how the nanoantenna affect to the Resonance Fluorescence Spectrum (RFS) of the hybrid system. This spectrum is proportional to the Fourier transformation of the steady-state correlation function $\lim _{t \rightarrow \infty}\left\langle E^{-}\left(r, t^{\prime}+t\right) \cdot E^{+}(r, t)\right\rangle$, where $E^{-}(r, t) / E^{+}(r, t)$ is the negative/positive frequency part of the radiation field in the far zone. The radiation field consists of a free-field operator and a source-field operator that is proportional to the atomic polarization operator [54]. Therefore, the RFS can be expressed in terms of the atomic correlation function

$$
S(\omega)=\Re\left[\int_{0}^{\infty} \lim _{t \rightarrow \infty}\left\langle E^{+}\left(t^{\prime}+t\right) \cdot E^{-}(t)\right\rangle e^{-i \omega t^{\prime}} d t^{\prime}\right],
$$

where $\Re[$ ] denotes the real part of the magnitude enclosed in square brackets, and $E^{+}(t)$ is the positive frequency part of the fluorescent field which in the far-field zone $\left(|\vec{r}| \gg c / \omega_{i j}, i=4,3, j=1,2\right)$ reads

$$
\begin{aligned}
\vec{E}^{+}(\vec{r}, t)= & \frac{\omega_{41}^{2}}{c^{2}|\vec{r}|} \vec{\mu}_{14} \sigma_{14}(t-|\vec{r}| / c)+\frac{\omega_{42}^{2}}{c^{2}|\vec{r}|} \vec{\mu}_{24} \sigma_{24}(t-|\vec{r}| / c) \\
& +\frac{\omega_{31}^{2}}{c^{2}|\vec{r}|} \vec{\mu}_{13} \sigma_{13}(t-|\vec{r}| / c)+\frac{\omega_{32}^{2}}{c^{2}|\vec{r}|} \vec{\mu}_{23} \sigma_{23}(t-|\vec{r}| / c),
\end{aligned}
$$


and $E^{-}(t)=\left(E^{+}(t)\right)^{\dagger}$. We will assume that $\omega_{41} \approx \omega_{42} \approx \omega_{31} \approx \omega_{32}$. We remind here that the following conditions hold: $\vec{\mu}_{14}=\vec{\mu}_{23}, \vec{\mu}_{13}=\vec{\mu}_{24}$, and $\vec{\mu}_{14} \perp \vec{\mu}_{24}$, whereas the direction of detection of the fluorescent field is perpendicular to the atomic dipole moments $\vec{\mu}_{14}$ and $\vec{\mu}_{23}$.

In writing Eq. (22) and in the rest of this Section, we abbreviate $\omega-\omega_{L}$ by $\omega$, but we should interpret $\omega$ as a frequency measured relative to the laser frequency $\omega_{L}$. We are only interested in the incoherent part of the RFS, which is given by

$$
S_{\text {inc }}(\omega)=\Re\left[\int_{0}^{\infty} \lim _{t \rightarrow \infty}\left\langle\Delta E^{+}\left(t^{\prime}+t\right) \cdot \Delta E^{-}(t)\right\rangle e^{-i \omega t^{\prime}} d t^{\prime}\right],
$$

where $\Delta E^{ \pm}\left(t^{\prime}\right)=E^{ \pm}\left(t^{\prime}\right)-\left\langle E^{ \pm}(\infty)\right\rangle$ stands for the deviation of the dipole polarization operator from its mean steady-state value. The calculation of $S_{\text {inc }}(\omega)$ requires the calculation of the two-time correlation function, which can be performed by means of the quantum-regression theorem [55, 54] (see Ref. [38] for details).
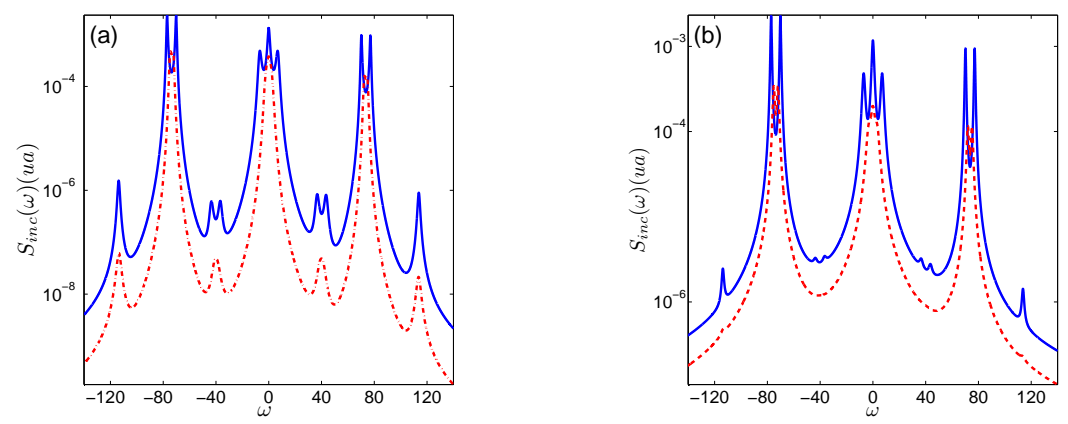

Figure 4: $S_{i n c}(\omega)$ versus $\omega$ for the isolated QD (solid line) and the hybrid system (dashed line) with gap $=65 \mathrm{~nm}$, and $\Omega_{c}^{0}=3.5 \gamma_{0}$. (a)/(b) RFS in the bare/dressed state basis.

Figure 4(a) presents the RFS computed in the bare basis of the QD in the absence of the nanoantenna (solid line) in the case that the Rabi frequency of the driving field is set to $\Omega_{c}^{0}=3.5 \gamma_{0}$. The central line exhibits a three peak structure. In addition, we can devise the appearance of five weak blue sidebands and five additional red sidebands. The latter being slightly greater than the former. It is worth mentioning that a Mollow-like quintuplet has been experimentally observed in the case of analyzing the RFS of InAs/GaAs quantum dots with a weak external field of $120 \mathrm{mT}$ applied in the Faraday 
geometry (see Ref. [56] for details). The RFS obtained for the current hybrid system is plotted with a dashed line and shows that either the central triplet and the red and blue closely lying doublets collapse into single lines: this result arises from the combined effect of the modification of the decay rates and the change of the driving field felt by the QD. We have shown in Fig. 2(a) that the presence of the nanoantenna alters the local density of states, which in turn manifests as an anysotropic modification of the decay rates. Such a change in the decay rates produces an enlargement of $\gamma_{42}^{p}=3.48 \gamma_{0}$, which in turn results in the broadening of the spectrum of the fluorescent photons. In addition, the plasmonic interaction also manifests by a field modification whose magnitude is given by $\left|F_{e}\right|<1$. We have seen in Fig. 2(b) that for a driving field polarized along the $X$ axis and for all the gap distances, the absolute value of $\left|F_{e}\right|$ is lesser than unity, indicating that the plasmonic interactions makes the driving field less effective in driving the QD transitions: $|1\rangle \leftrightarrow|4\rangle$ and $|2\rangle \leftrightarrow|3\rangle$. These two combined effects explain the origins of the collapse of the central triplet and the closely lying doublets into single peaks for the hybrid system. The use of the dressed state picture (see Ref. [38] for details) to compute the RFS in the current situation confirms the previous assertions, as depicted in Fig. 4(b): there we can appreciate that both the central line and the outermost doublets are clearly developed, whereas the innermost doublet and the outermost single line are scarcely appreciable in the dressed state picture. These features arise from the fact that the Rabi frequency of the driving field $\left(\Omega_{c}^{0}=3.5 \gamma_{0}\right)$ is sligthly greater than the free space decay rate $\left(\gamma_{0}\right)$ and is lesser than the value of $\gamma_{42}^{p}=3.48 \gamma_{0}$ for this particular situation, thus we expect the secular approximation used to derive the RFS in the dressed basis, whose validity relies on the fact that the Rabi frequency must be much greater than all the decay rates involved, not to be highly precise. Numerical computation carried out (not shown) reveals that the use of a Rabi frequency an order of magnitude larger than the one used to produce Fig. 4 results in the full development of the RFS and that the computation in both basis better agree, as expected. Finally, it is to be noted that measuring the spectral separation of the largest red sideband from the blue sideband it is possible to achieve photon emission across a frequency band of $\sim 172 \mathrm{GHz}$. This is nearly 225 times larger than the $0.76 \mathrm{GHz}$ spontaneous emission rate. This is by no mean an upper limit, but can be further increased by laser detuning, i.e., by setting $\Delta_{c} \neq 0$.

Now we investigate the plasmonic effect induced by the bowtie nanoantenne on the statistics of the fluorescent field. Thus, we focus on the second- 
order correlation function $g^{(2)}$, under stationary excitation which is given by

$$
g^{(2)}(\vec{r}, t, \vec{r}, t+\tau)=\frac{G^{(2)}(\vec{r}, t, \vec{r}, t+\tau)}{G^{(1)}(\vec{r}, t) G^{(1)}(\vec{r}, t+\tau)} .
$$

The first and second order correlation functions appearing in equation (25) can be expressed in terms of the positive and negative frequency parts of the electric field operators as

$$
\begin{aligned}
G^{(1)}(\vec{r}, t) & =<\vec{E}^{-}(\vec{r}, t) \vec{E}^{+}(\vec{r}, t)> \\
G^{(2)}(\vec{r}, t, \vec{r}, t+\tau) & =<\vec{E}^{-}(\vec{r}, t) \vec{E}^{-}(\vec{r}, t+\tau) \vec{E}^{+}(\vec{r}, t+\tau) \vec{E}^{+}(\vec{r}, t)>
\end{aligned}
$$

Magnitudes in Eq. (26) are evaluated under stationary conditions, thus equation (26) reduces to

$$
\begin{aligned}
G^{(1)}(\vec{r}, 0) & =<\vec{E}^{-}(\vec{r}, 0) \vec{E}^{+}(\vec{r}, 0)> \\
G^{(2)}(\vec{r}, 0, \vec{r}, \tau) & =<\vec{E}^{-}(\vec{r}, 0) \vec{E}^{-}(\vec{r}, \tau) \vec{E}^{+}(\vec{r}, \tau) \vec{E}^{+}(\vec{r}, 0)>.
\end{aligned}
$$

The normalized second order correlation function can be obtained from Eq. (26) as

$$
g^{(2)}(\vec{r}, t, \vec{r}, t+\tau)=\frac{G^{(2)}(\vec{r}, 0, \vec{r}, \tau)}{G^{(1)}(\vec{r}, 0) G^{(1)}(\vec{r}, 0)} .
$$

The use of the full positive frequency part of the fluorescent field given in Eq. (23) means that the detector could register fluorescent photons from transitions $|k\rangle \leftrightarrow|j\rangle(k=3,4$, and $j=1,2)$. However, the use of a linear polarizer between the hybrid system and the detector allows for a selective detection scheme. Thus, we will consider the use of an ideal linear polarizer whose transmission axis could be oriented along either the $X$ or the $Y$ axis. In view of the selection rules in Eq. (1), the positive part of the fluorescent field when the polarizer is oriented along the $X / Y$ axis should read as

$$
\begin{aligned}
& \vec{E}_{x}^{+}(\vec{r}, t)=\frac{\omega_{41}^{2}}{c^{2}|\vec{r}|} \vec{\mu}_{14} \sigma_{14}(t-|\vec{r}| / c)+\frac{\omega_{32}^{2}}{c^{2}|\vec{r}|} \vec{\mu}_{23} \sigma_{23}(t-|\vec{r}| / c), \\
& \vec{E}_{y}^{+}(\vec{r}, t)=\frac{\omega_{42}^{2}}{c^{2}|\vec{r}|} \vec{\mu}_{24} \sigma_{24}(t-|\vec{r}| / c)+\frac{\omega_{31}^{2}}{c^{2}|\vec{r}|} \vec{\mu}_{13} \sigma_{13}(t-|\vec{r}| / c) .
\end{aligned}
$$


The use of such selective detection scheme allow us to define the correlation functions

$$
\begin{aligned}
G_{k}^{(1)}(\vec{r}, 0) & =<\vec{E}_{k}^{-}(\vec{r}, 0) \vec{E}_{k}^{+}(\vec{r}, 0)>, \\
G_{k}^{(2)}(\vec{r}, 0, \vec{r}, \tau) & =<\vec{E}_{k}^{-}(\vec{r}, 0) \vec{E}_{k}^{-}(\vec{r}, \tau) \vec{E}_{k}^{+}(\vec{r}, \tau) \vec{E}_{k}^{+}(\vec{r}, 0)>, k=x, y(31) \\
g_{k}^{(2)}(\vec{r}, t, \vec{r}, t+\tau) & =\frac{G_{k}^{(2)}(\vec{r}, t, \vec{r}, t+\tau)}{G_{k}^{(1)}(\vec{r}, t) G_{k}^{(1)}(\vec{r}, t+\tau)},
\end{aligned}
$$

and $\vec{E}_{k}^{-}(\vec{r}, t)=\left[\vec{E}_{k}^{+}(\vec{r}, t)\right]^{\dagger}$ where superscript $\dagger$ stands for the hermitian conjugate.

The one-time correlation function in the case of full detection of the emitted fluorescent photons is easily evaluated and yields

$$
G^{(1)}(\vec{r}, 0)=G^{(1)}(\vec{r}, \tau) \propto\left(\gamma_{41}^{(0)}+\gamma_{42}^{(0)}\right) \rho_{44}^{S S}+\left(\gamma_{31}^{(0)}+\gamma_{32}^{(0)}\right) \rho_{33}^{S S},
$$

where the superscript $S S$ stands for "steady state" values of the corresponding magnitude, i.e., $\rho_{j j}^{S S}=\left\langle\sigma_{j j}(\infty)\right\rangle, j=3$, 4. In the case of using a polarizer for detection, the one-time correlation functions should reduce to

$$
\begin{aligned}
& G_{x}^{(1)}(\vec{r}, 0)=G_{x}^{(1)}(\vec{r}, \tau) \propto \gamma_{41}^{(0)} \rho_{44}^{S S}+\gamma_{32}^{(0)} \rho_{33}^{S S}, \\
& G_{y}^{(1)}(\vec{r}, 0)=G_{y}^{(1)}(\vec{r}, \tau) \propto \gamma_{42}^{(0)} \rho_{44}^{S S}+\gamma_{31}^{(0)} \rho_{33}^{S S} .
\end{aligned}
$$

The evaluation of the second order correlation function can be carried out with the aid of quantum regression theorem [55] and the optical Bloch Eq. (21). The details of the computation in Eq. (25) are given in Appendix A.

Figure 5(a) shows the second-order correlation function of the fluorescent field defined in Eq. (28) using the same data to produce Fig. 4. There we can appreciate that a strong bunching is obtained both for the case of the isolated QD (solid line) which is highly enhanced in the case of the the hybrid system (dashed-dotted line), with a clear shift in the peak of the burst of photons to a lower instant of time in the latter case. This effect is further confirmed by taking a look at the short-time behavior of the $g^{(2)}(\tau)$ depicted at the the inset of Fig. 5(a), which reveals that the crossing time from antibunching to bunching shortens for the hybrid system. The closeness of the peak of the burst of photons to the characteristic time in the case of the hybrid system suggest us to use it as an indirect indication that the system is nearly initialized, and could be used by experimentalist to ascertain such spin initialization. 

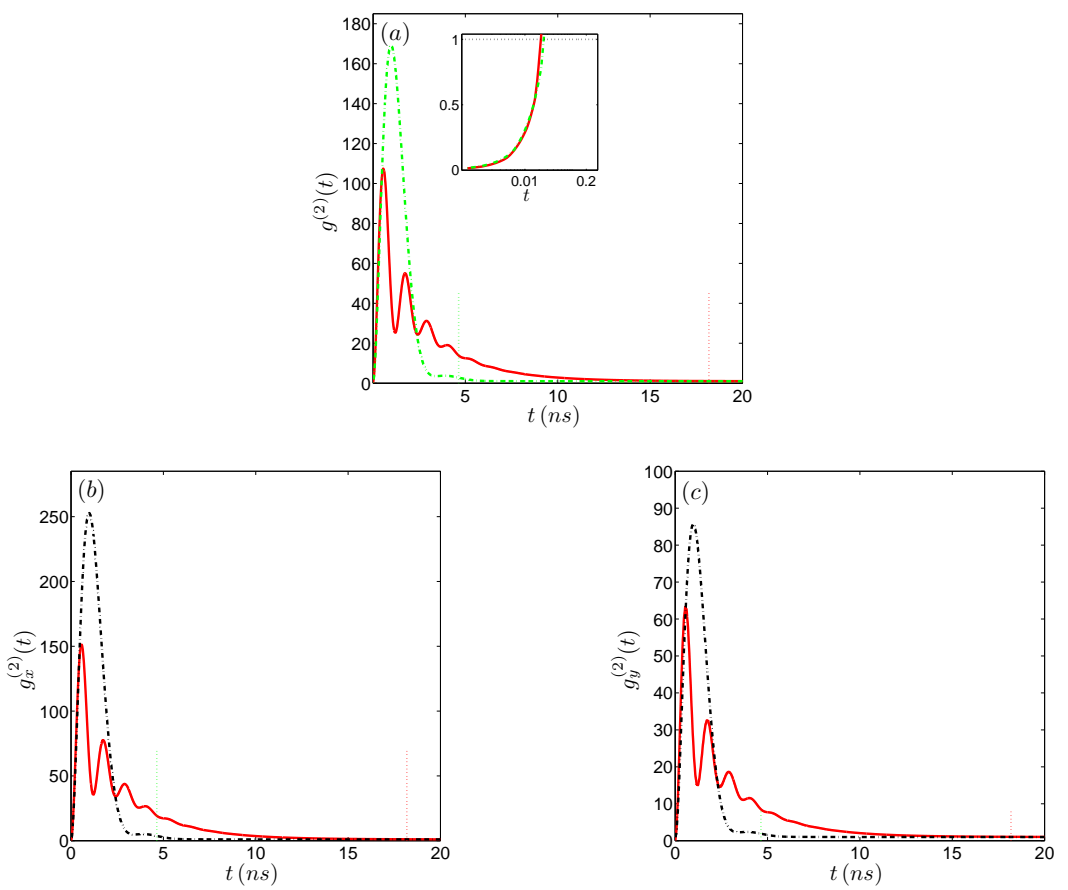

Figure 5: (a) Time evolution of $g^{(2)}(\tau)$ of an isolated QD (solid line) and the hybrid system (dashed-dotted line). (b)/(c) Time evolution of $g_{x}^{(2)}(\tau) / g_{y}^{(2)}(\tau)$ line) when using a polarizer whose transmission axis is oriented along the $X / Y$ axis, respectively. The vertical dotted lines indicate the corresponding characteristic time $T_{0}$ ordered from left to right for the hybrid system and the isolated QD. The Rabi frequency used is $\Omega_{c}^{0}=3.5 \gamma_{0}$ and the gap distance is set to $65 \mathrm{~nm}$.

The use of a selective polarization detection of the photons of the fluorescent field, reveals a distinctive behavior depending on the orientation of the linear polarizer. The results for the case of a polarizer oriented along the $X / Y$-axis are presented in Fig. 5(b)/(c). Note that in the case of setting the polarizer along the $X$-axis results in a bunching signal whose peak is larger for for the hybrid system (dashed-dotted line) than that obtained for the isolated QD (solid line). However, in the case of using a polarizer along the $Y$ axis the peak of the bunching signal for the hybrid system exhibits a sevenfold enhancement. This result arises since we are aware that transition $|4\rangle \leftrightarrow|2\rangle$ becomes highly enhanced in comparison with transision $|4\rangle \leftrightarrow|1\rangle$ as a result of the plasmonic interaction. Note again that the peak of the bunching signal becomes closest to the initialization time (see vertical 
dotted lines in Fig. 5). In addition, the crossing time from antibunching to bunching is strongly accelerated in the case of using a linear polarizer along the $Y$ axis as depicted in the inset of Fig. 5(c).

\section{Conclusions}

In this work we present a description of the influcence of exciton-plasmon interaction in a QD-bowtie nanoantenna hybrid system on spin initialization. The QD is modeled as a four level-like atomic system and the properties of the nanoantenna are taken into account by using the boundary element method to account either for the enhancement of the Rabi frequency of the driving field and for the modifications of the decay rates of the QD. The influence of the gap size of the nanoantenna on such parameters is analyzed in detail. In view of the geometric configuration of the nanoantenna and the orientation of the dipole moments of the QD, we show that the anysotropic enhancement of the decay rates results in a fast spin initialization. A new definition of the characteristic time is proposed which accounts for the time required to achieve a certain level of population of the target level with regard to the maximum level attainable in the steady state. This results in the existence of an interval of Rabi frequencies which allows for such initialization within a certain level of tolerance.

We show that the anysotropic modification of the decay rates results in dramatic changes of the RFS of the hybrid system. We have predicted that the lines of the RFS can be broadened for the range of Rabi frequencies of interest for spin initialization. The modifications of the RFS predicted in this work, represents a way for testing experimentally how the exciton-plasmon interaction modifies the properties of the spontaneous emission of the decay pathways in a positively charged QD. Most interestingly, we have analyzed how the exciton-plasmon interaction modifies the second order correlations of the fluorescent fields, showing that the peak of bunching photons is obtained close to the instant of time where the system is initialized. The use of selective detection of the fluorescent photon has been analyzed and shows a high enhancement for the peak of the burst signal in the case of setting the polarizer axis along paralell to the axis of the driving field.

Finally, it should be stated that, although speculative, the hybrid system here investigated can be implemented in realistic QD-MNP systems. Recently, hybrid structures consisting of self-assembled QDs has been grown and covered with metall nanocrystals [57]. In addition, we would like to 
draw the attention to very recent works where the controlled coupling of a single epitaxial quantum dot to a plasmonic nanoantenna has been demonstrated [58, 59]. Thus, the structure modeled here could be fabricated using available nano-technologies for the growth and the precise positioning of the involved elements. The current approach represents a fast way to obtain the spin initialization with high fidelity, which is required for quantum information processing, while taking advantage of a positively charged QD where the dephasing due to nuclear spins is minimized. 


\section{Appendix A. Intensity-intensity correlation}

The evaluation of the second order correlation function can be carried out with the aid of quantum regression theorem [55] and the optical Bloch equations (21). To this end we define the column vectors

$$
\begin{aligned}
U^{k i j k}(\tau)=[ & \left\langle\sigma_{k i}(\infty) \sigma_{41}(\tau) \sigma_{j k}(\infty)\right\rangle,\left\langle\sigma_{k i}(\infty) \sigma_{14}(\tau) \sigma_{j k}(\infty)\right\rangle \\
& \left\langle\sigma_{k i}(\infty) \sigma_{42}(\tau) \sigma_{j k}(\infty)\right\rangle,\left\langle\sigma_{k i}(\infty) \sigma_{24}(\tau) \sigma_{j k}(\infty)\right\rangle \\
& \left\langle\sigma_{k i}(\infty) \sigma_{31}(\tau) \sigma_{j k}(\infty)\right\rangle,\left\langle\sigma_{k i}(\infty) \sigma_{13}(\tau) \sigma_{j k}(\infty)\right\rangle \\
& \left\langle\sigma_{k i}(\infty) \sigma_{32}(\tau) \sigma_{j k}(\infty)\right\rangle,\left\langle\sigma_{k i}(\infty) \sigma_{23}(\tau) \sigma_{j k}(\infty)\right\rangle \\
& \left\langle\sigma_{k i}(\infty) \sigma_{21}(\tau) \sigma_{j k}(\infty)\right\rangle,\left\langle\sigma_{k i}(\infty) \sigma_{12}(\tau) \sigma_{j k}(\infty)\right\rangle \\
& \left\langle\sigma_{k i}(\infty) \sigma_{43}(\tau) \sigma_{j k}(\infty)\right\rangle,\left\langle\sigma_{k i}(\infty) \sigma_{34}(\tau) \sigma_{j k}(\infty)\right\rangle \\
& \left\langle\sigma_{k i}(\infty) \sigma_{44}(\tau) \sigma_{j k}(\infty)\right\rangle,\left\langle\sigma_{k i}(\infty) \sigma_{33}(\tau) \sigma_{j k}(\infty)\right\rangle \\
\langle & \left.\left.\sigma_{k i}(\infty) \sigma_{22}(\tau) \sigma_{j k}(\infty)\right\rangle\right]^{T}(k=3,4, i, j=1,2),
\end{aligned}
$$

where superindex $T$ stands for transpose. The following identity $\left\langle\sigma_{k i}\right\rangle=\rho_{i k}$ holds. According to the quantum regression theorem, for $\tau>0$, the vectors $U^{k i j k}(\tau)$ satisfy

$$
\frac{d U^{k i j k}(\tau)}{d \tau}=M U^{k i j k}(\tau),+B<\sigma_{k i}(\infty) \sigma_{j k}(\infty)>(k=3,4, i, j=2,(3), 2)
$$

$M$ being the $15 \times 15$ matrix of the coefficients of equation (21), and $B$ is the column vector

$$
B=\left[-i \Omega_{c}^{*}, i \Omega_{c}, 0,0,0,0,0,0,0,0,0,0,0,0,0\right]^{T} .
$$

We can determine the values of $U^{k i j k}(\tau)$ by integrating equation (31) with proper initial conditions which are determined by computing $U^{k i j k}(\tau=0)$ in terms of the steady state values of populations and coherences.

\section{Acknowledgments}

This work has been supported by Project no. FIS2013-41709-P (MICINN) from Spain.

[1] J.M. Elzerman, R.Hanson, L.H. Willems van Beveren, B. Witkamp, L.M.K. Vandersypen, L.P. Kouwenhoven, Nature 430 (2004) 431. 
[2] D. Heiss, S. Schaeck, H. Huebl, M. Bichler, G. Abstreiter, J.J. Finley, D.V. Bulaev, D. Loss, Phys. Rev. B 76 (2007) 241306.

[3] A. Zrenner E. Beham, S. Stufler, F. Findeis, M. Bichler, G. Abstreiter, Nature 418 (2002) 612.

[4] X. Li,Y. Wu, D. Steel, D. Gammon, T.H. Stievater, D.S. Katzer, D. Park, C. Piermarocchi, L.J. Sham, Science 301 (2003) 809.

[5] A. Imamoglu, D.D. Awschalom, G. Burkard, D.P. DiVincenzo, D. Loss, M. Sherwin, A. Small, Phys. Rev. Lett. 83 (1999) 4204.

[6] G. Burkard, D. Loss, D.P. DiVincenzo. Phys. Rev. B 59 (1999) 2070.

[7] M. Atatüre J. Dreiser, A. Badolato, A. Högele, K. Karrai, A. Imamoglu, Science 312 (2006) 551.

[8] D.V. Bulaev, D. Loss. Phys. Rev. Lett. 95 (2005) 076805.

[9] D. Brunner, B.D. Gerardot, P.A. Dalgarno, G. Wüst, K. Karrai, N.G. Stoltz, P.M. Petroff, R.J. Warburton, Science 325 (2009) 70.

[10] K.G. Lagoudakis, K.A. Fischer, T. Sarmiento, K. Mueller, Jelena Vučković, Phys. Rev. B 90 (2014) 121402.

[11] C. Emary, X. Xu, D.G. Steel, S. Saikin, L.J. Sham, Phys. Rev. Lett. 98 (2007) 047401.

[12] V. Loo, L. Lanco, O. Krebs, P. Senellart, P. Voisin, Phys. Rev. B 83 (2011) 033301.

[13] M.A. Antón, F. Carreño, S. Melle, O.G. Calderón, E. Cabrera-Granado, M.R. Singh, Phys. Rev. B 87 (2013) 195303.

[14] K.R. Li, M.I. Stockman, D.J. Bergman, Phys Rev Lett. 91 (2003) 227402 .

[15] S.A. Maier. Plasmonics: Fundamentals and Applications. 2007 Springer US.

[16] W. Zhang, A.O. Govorov, G.W. Bryant, Phys. Rev. Lett. 97 (2006) 146804 . 
[17] R. D. Artuso, G.W. Bryant, Nano Lett. 8 (2008) 2106.

[18] K.T. Shimizu, W.K. Woo, B.R. Fisher, H.J. Eisler, M. G. Bawendi, Phys. Rev. Lett. 89 (2002) 117401.

[19] A.O. Govorov, G.W. Bryant, W. Zhang, T. Skeini, J. Lee, N.A. Kotov, J.M. Slocik, R.R. Naik, Nano Lett. 6 (2006) 984.

[20] J.D. Cox, M.R. Singh, C. von Bilderling and A.V. Bragas, Adv. Opt. Mat. 1 (2013) 460.

[21] A. Manjavacas, F.J. García de Abajo, P. Nordlander, Nano Lett. 11 (2011) 2318.

[22] A. Manjavacas, P. Nordlander, F.J. García de Abajo, ACS Nano 2 (2012) 1724 .

[23] F.H.L. Koppens, D.E. Chang, F.J. García de Abajo, Nano Lett. 11 (2011) 3370.

[24] V.V. Klimov, M. Ducloy, V.S. Letokhov, Eur. Phys. J. D 20 (2002) 133.

[25] E. Paspalakis, S. Evangelou, V. Yannopapas and A. F. Terzis, Phys. Rev. A 88 (2013) 053832.

[26] E. Paspalakis, S. Evangelou, S. G. Kosionis and A. F. Terzis, J. Appl. Phys. 115 (2014) 083106.

[27] S. Evangelou, V. Yannopapas and E. Paspalakis, J. Mod. Optics 61 (2014) 1458.

[28] S. Link, M.A. El-Sayed, J. Phys. Chem. B 103 (1999) 8410.

[29] C. Racknor, M.R. Singh, Y. Zhang, D.J.S. Birch and Y. Chen, Methods Appl. Fluoresc. 2 (2014) 015002.

[30] C.J. Murphy, T.K. Sau, A. Gole, C.J. Orendorff, MRS Bull. 30 (2005) 349.

[31] Y. Sun, Y. Xia, Science 298 (2002) 2176.

[32] L.J. Sherry, S.H. Chang, G.C. Schatz, R.P.V. Duyne, B.J. Wiley, Y. Xia, Nano Lett. 5 (2005) 2034. 
[33] C.L. Nehl, H. Liao, J.H. Hafner, Nano Lett. 6 (2006) 683.

[34] H. Xu, H.E.J. Bjerneld, M. Kall, M.L. Borjesson, Phys. ReV. Lett. 83 (1999) 4357.

[35] J.B. Lassiter, J. Aizpurua, L.I. Hernandez, D.W. Brandl, I. Romero, S. Lal, J.H. Hafner, P. Nordlander, N.J. Halas, J. Nano Lett. 8 (2008) 1212 .

[36] A.E. Schlather, N. Large, A.S. Urban, P. Nordlander, and N.J. Halas Nano Lett. 13 (2013) 3281.

[37] S. Thongrattanasiri, F.J. García de Abajo, Phys. Rev. Lett. 110, (2013) 187401.

[38] F. Carreño, M.A. Antón, F. Arrieta-Yáñez Phys. Rev. B 88 (2013) 195303.

[39] D. Brunner, Ph.D. Thesis, Herior-Watt University, 2010, http://hdl.handle.net/10399/2350.

[40] B.D. Gerardot, D. Brunner, P.A. Dalgarno, P. Öhberg, S. Seidl, M. Kroner, K. Karrai, N. G. Stoltz, P.M. Petroff, R.J. Warburton, Nature 451 (2008) 441.

[41] M. Kroner, K.M. Weiss, B. Biedermann, S. Seidl, A.W. Holleitner, A. Badolato, P.M. Petroff, P. Öhberg, R.J. Warburton, K. Karrai, Phys Rev. B 78 (2008) 075429.

[42] E.D. Kim, K. Truex, X. Xu, B. Sun, D.G. Steel, A.S. Bracker, D. Gammon, L.J. Sham, Phys. Rev. Lett. 104 (2010) 167401.

[43] D. Press, T.D. Ladd, B. Zhang, Y. Yamamoto, Nature 456 (2008) 218.

[44] H.T. Dung, L. Knoll, D.G. Welsch, Phys Rev. A 66 (2002) 063810.

[45] G.X. Li, F.L. Li, S.Y. Zhu, Phys. Rev. A 64 (2001) 013819.

[46] Y. Yang, J. Xu, H. Chen, S. Zhu, Phys. Rev. Lett. 100 (2008) 043601.

[47] G.-X. Li, J. Evers, C. H. Keitel, Phys. Rev. B 80 (2009) 045102.

[48] J.-P. Xu and Y.-P. Yang, Phys. Rev. A 81 (2010) 013816. 
[49] S. Evangelou, V. Yannopapas, E. Paspalakis, Phys. Rev. A 86 (2012) 053811 .

[50] W.L. Barnes, J. Mod. Opt. 45 (1998) 661.

[51] U. Hohenester, A. Trügler, Comp. Phys. Commun. 183 (2012) 370.

[52] P.B. Johnson, R. W. Christy, Phys. Rev. B 6 (1972) 4370.

[53] O. Ridolfo, N. Di Stefano, N. Fina, R. Saija, and S. Savasta, Phys. Rev. Lett. 105 (2010) 263601.

[54] M.O. Scully M.S. Zubairy, Quantum Optics, 1997 Cambridge University Press.

[55] M. Lax, Phys. Rev. 172 (1968) 350.

[56] A.N. Vamivakas, Y. Zhao, Chao-Yang Lu, M. Atatüre, Nat. Phys. 5 (2009) 198.

[57] A. Urbańczyk, G.J. Hamhuis, R. Nötzel, Appl. Phys. Lett. 96 (2010) 113101.

[58] M. Pfeiffer, K. Lindfors, C. Wolpert, P. Atkinson, M. Benyoucef,A. Rastelli, O. G. Schmidt, H. Giessen, M. Lippitz, Nano Lett. 10 (2010) 4555 .

[59] M. Pfeiffer, K. Lindfors, P. Atkinson, A. Rastelli, O.G. Schmidt, H. Giessen, M. Lippitz, Phys. Stat. Sol. 249 (2012) 678. 